\title{
Clinical Characteristics of Patients with Cryptogenic Hepatocellular Carcinoma in a Hepatitis B Virus-Endemic Area
}

\author{
Hee-Won Kwak ${ }^{a}$ Joong-Won Park ${ }^{a}$ Young Hwan Koh ${ }^{a}$ \\ Ju Hee Lee Ami Yub $^{\mathrm{b}}$ Byung-Ho Namb \\ ${ }^{a}$ Center for Liver Cancer, ${ }^{b}$ Cancer Biostatistics Branch, National Cancer Center, Goyang, \\ Republic of Korea
}

\section{Key Words}

Anti-HBc antibodies · Cryptogenic · Hepatocellular carcinoma - Non-alcoholic fatty liver disease $\cdot$ Non-B non- $C$ non-alcohol

\begin{abstract}
Objectives: Chronic hepatitis B virus (HBV) infection is the most common cause of hepatocellular carcinoma (HCC) in South Korea, but a high prevalence of metabolic diseases may result in increases in the incidence of cryptogenic HCC ( $\mathrm{cHCC}$ ). We studied characteristics of the cHCC in a single-center cohort. Methods: A cohort of 1,784 HCC patients newly diagnosed and treated at the National Cancer Center, Korea, between 2004 and 2009 was reviewed and analyzed. Results: The cause of HCC was categorized as CHCC, HBV, hepatitis C virus (HCV), or alcohol. Overall, 162 (9.1\%) patients of the HCC cohort had CHCC, and their mean age was 61.9 years. The median survival of CHCC patients was 24.7 months, which was the second shortest among the four groups after HBV HCC. CHCC patients had the largest tumor size (mean $7.4 \mathrm{~cm}$ ) and the second highest proportion of poor prognostic factors such as the proportion of poorly defined tumors and extrahepatic spread in imaging studies. CHCC patients had better survival than HBV HCC patients according to multivariate analysis. Among CHCC patients, 137 (84.6\%) had anti-HBc IgG antibodies, but this sub-group had different clinical features to those of HBV HCC patients. The body mass index (BMI) and hyperglycemia and hypercholesterolemia levels in $\mathrm{CHCC}$ patients were similar to those in HCV and alcoholic HCC patients. Conclusions: Anti-HBC IgG antibodies were present in most $\mathrm{CHCC}$ patients, but CHCC patients had better survival than HBV HCC patients on multivariate analysis. However,
\end{abstract}


cHCC patients had a larger mean tumor size and more aggressive tumor characteristics than HCV HCC or alcoholic HCC patients did. It is hoped that this study will contribute to a better understanding of $\mathrm{CHCC}$ in HBV-endemic areas.

Copyright $\odot 2015$ S. Karger AG, Basel

\section{Introduction}

Hepatocellular carcinoma (HCC) may result from liver cirrhosis of various causes, including hepatitis B virus (HBV) infection, hepatitis C virus (HCV) infection, chronic alcohol consumption [1, 2], non-alcoholic fatty liver disease (NAFLD) [3-8], and unknown causes [9]. Although the incidence of HCC varies in different localities, HBV and HCV infections are the most common causes of HCC [10]. However, HBV vaccination programs and the prevention of iatrogenic infections (such as HCV screening of transfused blood) have reduced the incidence of HCC of viral causes, whereas the proportion of HCC of non-viral cause has, as a result, relatively increased [11]. The majority of (cHCC) cases in the United States are caused by NAFLD, a hepatic manifestation of the metabolic syndrome [12]. NAFLD-associated HCC accounts for more than half of patients with non-HBV, non-HCV, non-alcoholic (NBNCNA) cHCC cases in some reports $[5,13]$. Because the proportion of the population with metabolic syndrome has increased, NAFLD has also increased recently in Asian as it has in Western countries [14], and now it is present in $5 \%-40 \%$ of the general population $[15,16]$. NAFLD can progress to non-alcoholic steatohepatitis (NASH), cirrhosis, and HCC [3-7]. In the United States, among patients older than 60 years, NAFLD appears to be responsible for as much $\mathrm{HCC}$ as HCV is [17].

There are a few reports on the characteristics of cirrhosis according to cause [18-20]; however, such comparative studies of HCC are rare [4, 21]. In South Korea, the incidence of cryptogenic, or NBNCNA, HCC has reportedly increased from $2.3 \%$ to $12.2 \%$ [21-24]. Therefore, there is a need for better understanding of cHCC. In this cohort study, we examined the clinical characteristics, metabolic factors, and prognostic factors in patients with cHCC compared with those with HCC from other causes in a HBV-endemic area.

\section{Materials and Methods}

\section{Patients and Classification of HCC According to Cause}

A cohort of 1,972 patients newly diagnosed with HCC and treated at the National Cancer Center hospital, Korea, between January 2004 and December 2009 was investigated for this study [25]. The patients were enrolled prospectively, and relevant clinical data and tumor characteristics were extracted retrospectively from their medical records. The patients were followed up until March 2012. HCC was diagnosed on the basis of the guidelines of the Korea Liver Cancer Study Group/NCC, Korea [26, 27]. A total of 185 patients with inadequate or missing metabolic values and 3 rare cases (one case each of BuddChiari syndrome, primary biliary cirrhosis, and autoimmune hepatitis) were excluded, and so 1,784 HCC patients were finally enrolled in this study.

In HCC patients with positive HBsAg or anti-HCV test results, HBV or HCV infection, respectively, was considered to be the cause of HCC (HBV HCC and HCV HCC, respectively), regardless of any history of alcohol consumption. Serum HBsAg and anti-HCV were determined with chemiluminescent immunoassays using an Architect HBsAg QT (Abbott Laboratories, Abbott Park, IL, USA) and Advia Centour XP Immunoassay system and Advia Centaur HCV (Siemens, Germany) assays. HCC patients who were negative for both HBsAg and anti-HCV, but had a history of chronic alcohol abuse, were classified as alcoholic HCC. The history of chronic alcohol abuse was checked by a review of medical records, including questionnaires. HCC patients who were negative for HBsAg and anti-HCV and who had no history of chronic alcohol abuse were classified as having cHCC. 
We defined cHCC as NBNCNA HCC, but many of the cHCC patients might have had a past infection with HBV. Even though HBsAg appeared negative, the existence of anti-HBc from an occult or past infection might have influenced the characteristics of HCC [28]. Therefore, we performed additional subanalyses according to the presence of anti-HBc antibody in cHCC patients. Moreover, the presence of fatty liver, which is one of the crucial findings of cHCC, was determined with imaging studies using ultrasound, computed tomography (CT), or magnetic resonance imaging (MRI).

\section{Variables Analyzed}

The Modified Union for International Cancer Control (mUICC) stages were used for tumor staging [29], and the Barcelona Clinic for Liver Cancer (BCLC) staging system was used for clinical staging [30]. The Child-Pugh and Model for End-stage Liver Disease (MELD) scoring systems were used for clinical diagnosis and classification of the severity of liver dysfunction [31]. The tumor type (i.e., well or poorly defined HCC), the presence of cirrhosis or fatty liver, portal vein thrombosis, and extrahepatic spread were confirmed by initial CT imaging studies at the first visit. Additionally, to establish the severity of liver fibrosis, we measured platelet counts and the aspartate aminotransferase to platelet ratio (APRI), an index that reflects liver fibrosis $[13,32]$.

We retrospectively analyzed survival, tumor stage, and characteristics that might affect the prognosis of HCC patients according to cause. We also compared the initial treatments, which included liver transplantation, radiofrequency ablation therapy (RFA), liver resection, conventional transarterial chemoembolization (CTACE) therapy, external beam radiation therapy, systemic cytotoxic chemotherapy, sorafenib (molecular-targeted therapy), and conservative management.

In addition, we reviewed the clinical records of HCC patients with respect to clinical and laboratory variables such as body mass index (BMI), blood pressure, fasting glucose values, total serum cholesterol values, and liver function tests [such as serum aspartate aminotransferase (AST) and alanine aminotransferase (ALT)]. We also checked for fatty liver in the initial CT imaging studies.

Hypertension was defined as systolic blood pressure $>140 \mathrm{mmHg}$ or diastolic blood pressure $>90$ $\mathrm{mmHg}$ at first visit or a previous diagnosis of hypertension. Hyperglycemia was defined as fasting glucose level $>126 \mathrm{mg} / \mathrm{dL}$ [33] or a previous diagnosis of diabetes mellitus (DM). Hypercholesterolemia was defined as total cholesterol $>240 \mathrm{mg} / \mathrm{dL}$ [34]. The study protocol was approved by the Institutional Review Board of the National Cancer Center, Korea [NCCNCS-13-821].

\section{Statistical Analyses}

The Kaplan-Meier method and the Cox proportional hazard model were used for survival analysis. Chi-square tests were employed to compare categorical variables. Independent t-tests were used to define whether there was a statistically significant difference between the means in two unrelated groups. For variables not following a normal distribution (e.g., ASL/ALT), an analysis of variance test was also performed. All statistical analyses were performed with STATA version 10.0 (Stata Corp, College Station, TX, USA), and values of $\mathrm{p}<0.05$ were considered statistically significant.

\section{Results}

\section{Survival and Clinicopathologic Characteristics}

A total of 1,784 HCC patients were classified according to the cause of HCC. The number of cases of cHCC, HBV HCC, HCV HCC, and alcoholic HCC were 162 (9.2\%), 1,320 (74.0\%), 167 (9.4\%), and 135 (7.5\%), respectively. Comparisons of overall survival and clinicopathologic characteristics between CHCC patients and patients with HCC due to other causes are presented in tables 1, 2 and fig. 1. The 1-year, 3-year, and 5-year survival rates of cHCC were $61.7 \%$, $42.2 \%$, and $35.3 \%$, respectively, and no significant differences were found between these survival rates and those of HBV HCC, HCV HCC, or alcoholic HCC (fig. 1). Median survival was 24.7 months for cHCC, which was the second shortest after that of HBV HCC (18.6 months). cHCC patients [hazard ratio $(\mathrm{HR})=1$ ] had better survival than HBV HCC patients $(\mathrm{HR}=1.251$ ) on multivariate analysis (table 2).

The mean age at diagnosis of cHCC patients (61.9 years) was less than that for HCV HCC patients (66.1 years) and greater than that for HBV HCC patients $(53.7$ years) (both $\mathrm{p}<0.001$ ). 


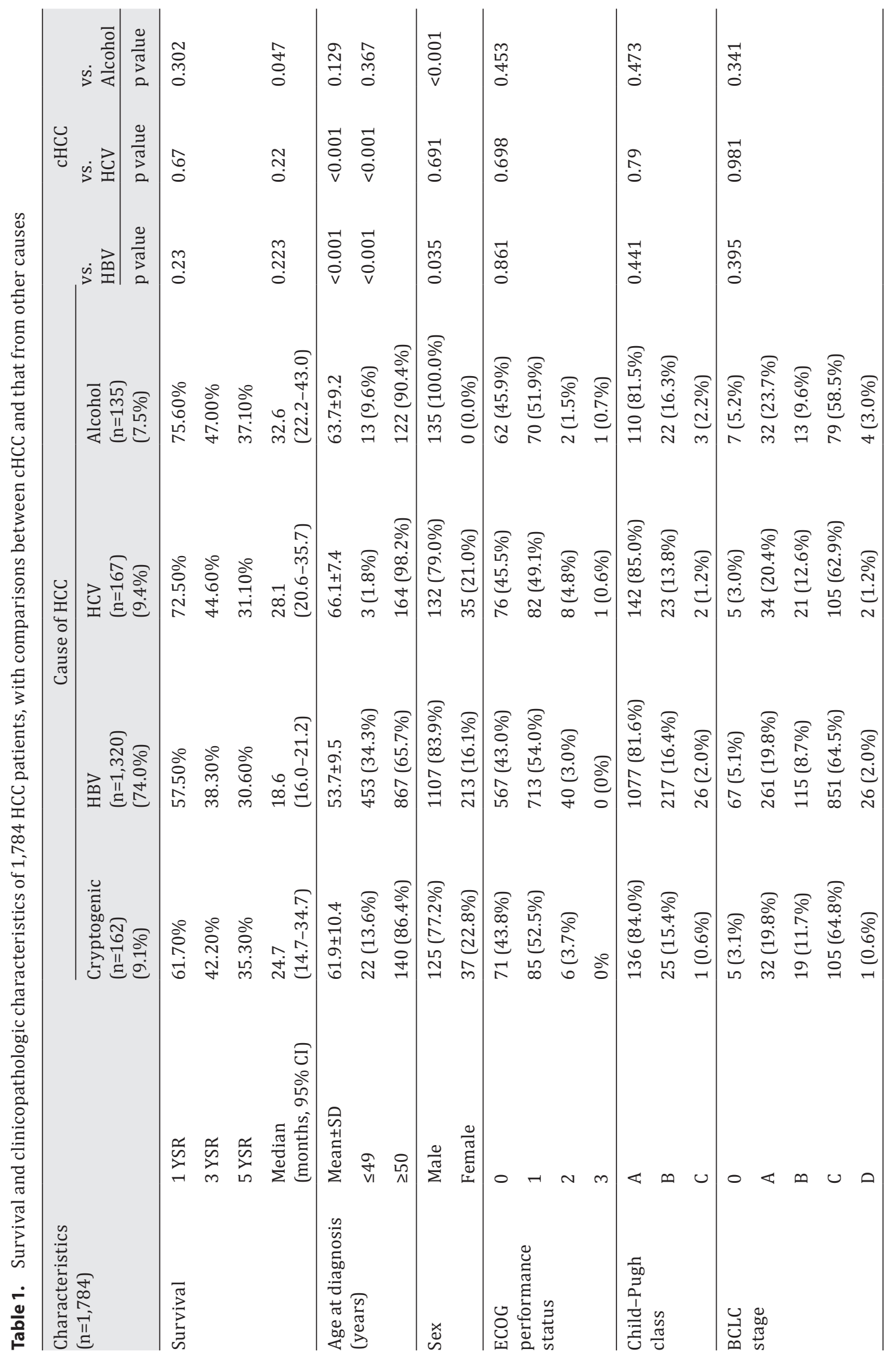




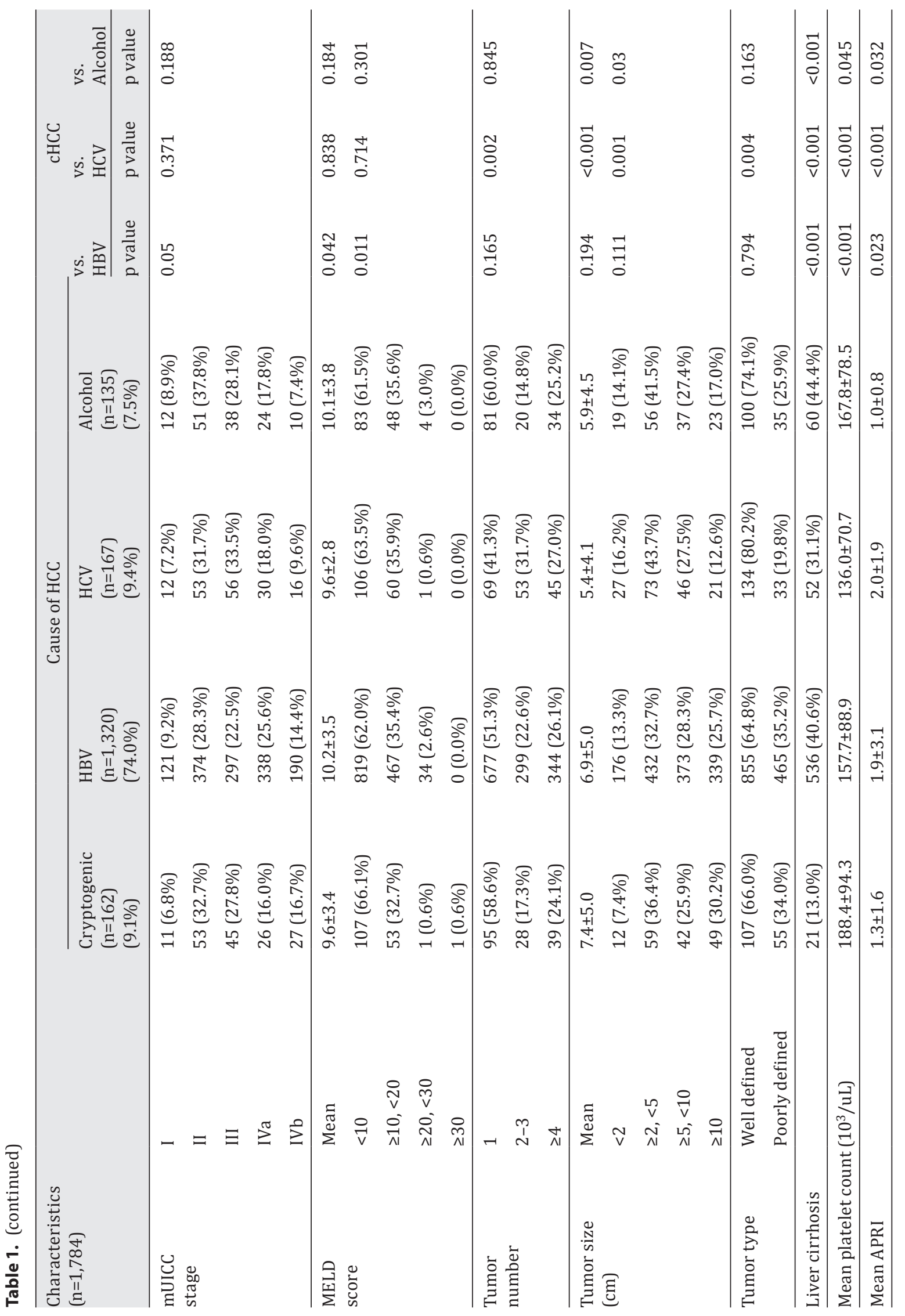




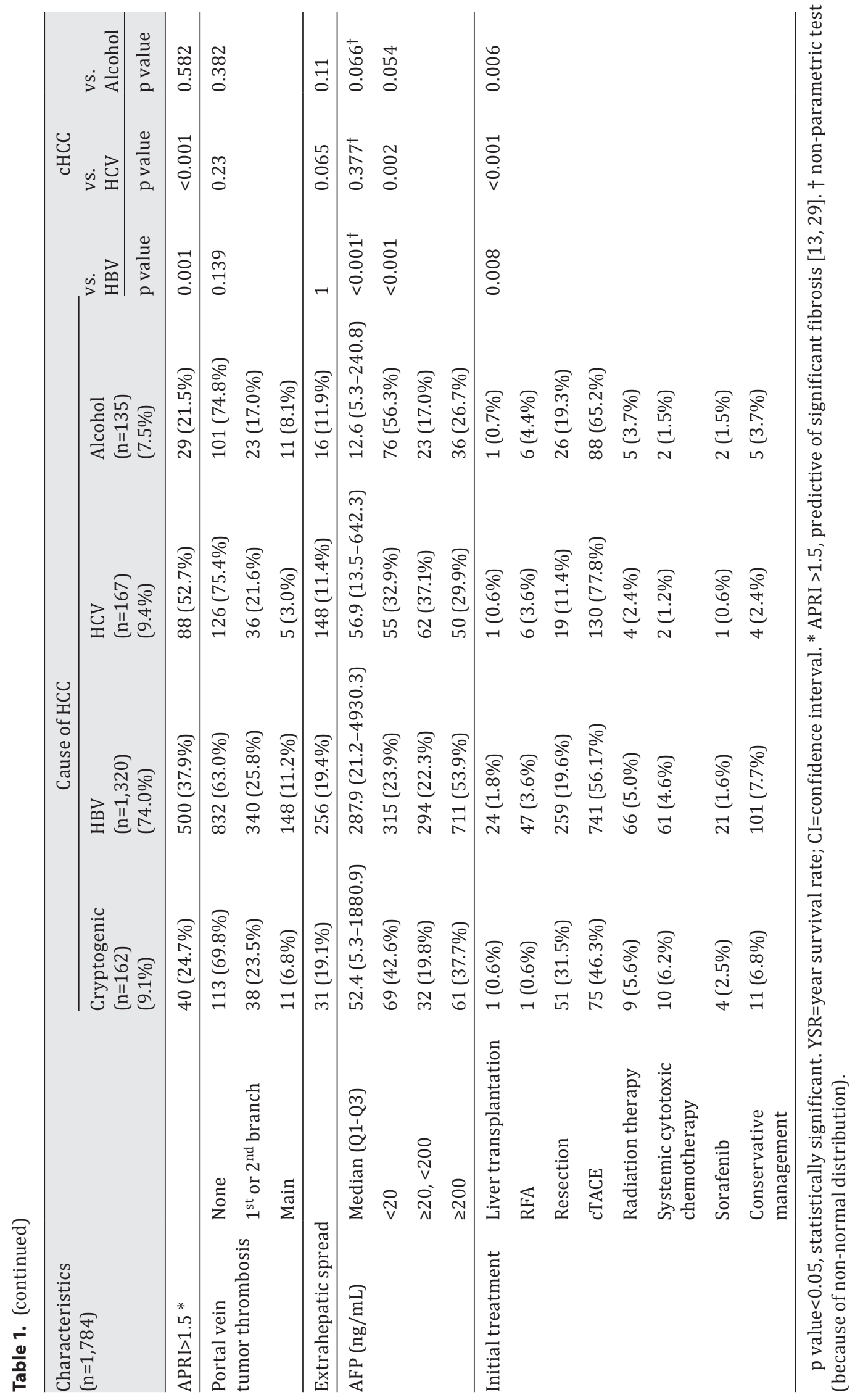


Table 2. Comparison of survival rates between $\mathrm{CHCC}$ and HCC from other causes (HR of death through multivariate analysis)

\begin{tabular}{llllll}
\hline & & p value & HR & $95 \%$ CI & \\
\hline Cause of HCC & Cryptogenic & & 1 & & \\
& HBV & 0.0383 & 1.251 & 1.012 & 1.547 \\
& HCV & 0.9256 & 0.987 & 0.747 & 1.304 \\
& Alcohol & 0.9211 & 1.015 & 0.756 & 1.363 \\
\hline
\end{tabular}

cHCC had the largest proportion of female patients (22.8\%) compared with HBV HCC $(16.1 \%$, $\mathrm{p}=0.035)$, HCV HCC $(21.0 \%, \mathrm{p}=0.691)$, and alcoholic HCC $(0 \%, \mathrm{p}<0.001)$. The performance status, Child-Pugh class, and BCLC stage were not different among the groups of HCC patients. The mUICC stage of cHCC at diagnosis was lower than that of HBV HCC $(p=0.050)$. A MELD score $<10$ was most frequent $(66.1 \%)$ in $\mathrm{CHCC}$, and the MELD score distribution of cHCC had a higher proportion of lower stages than that of HBV HCC $(p=0.011)$.

cHCC patients had the second-highest proportion of single hepatic lesions (58.6\%), after alcohol HCC $(60.0 \%)$, and the number of cHCC nodules was smaller than that of HCV HCC $(\mathrm{p}=0.002)$. cHCC patients had the largest tumors (mean $7.4 \mathrm{~cm} ; 30.2 \%>10 \mathrm{~cm}$ ) among HCC patients, and the tumor size distribution showed a smaller proportion of tumors $<2 \mathrm{~cm}$ than for HCV and alcoholic HCC ( $\mathrm{p}<0.05)$. In addition, as determined by CT imaging, cHCC patients had the second-highest proportion of poorly defined tumors (34.0\%), after HBV HCC (35.2\%), and significantly more than that of HCV HCC $(19.8 \%, p=0.004)$.

Initial diagnostic CT imaging studies revealed that $\mathrm{CHCC}$ patients had the lowest prevalence of cirrhosis (13.0\%), followed by HCV HCC (31.1\%), HBV HCC (40.6\%), and alcoholic HCC $(44.4 \%)$ patients. cHCC patients had the highest mean platelet count $\left(188.4 \times 10^{3} /\right.$ $\mu \mathrm{L})$. cHCC patients also had lower mean APRI values (1.3) than patients with HBV HCC (1.9, $\mathrm{p}=0.023)$ and HCV HCC $(2.0, \mathrm{p}<0.001)$. The proportion of cryptogenic patients with APRI $>1.5$, which is a predictive of significant fibrosis [13,35], was lower than that of HBV HCC patients (24.7\% vs. $37.9 \%, p=0.001)$ and HCV HCC patients $(24.7 \%$ vs. $52.7 \%$, respectively, $\mathrm{p}<0.001$ ).

cHCC patients had the second-highest frequency of portal vein tumor invasion and extrahepatic spread after HBV HCC (30.3\% vs. $37.0 \%$ and $19.1 \%$ vs $19.4 \%$, respectively, $p>0.05)$.

cHCC had the second highest number of patients with normal $\alpha$-fetoprotein (AFP) values (42.6\%), after those of alcoholic HCC (56.3\%). Median AFP levels were significantly different between cHCC and HBV HCC (52.4 vs. $287.9 \mathrm{ng} / \mathrm{mL}, \mathrm{p}<0.001)$. Resection was performed at a higher proportion of all treatment modalities for cHCC (31.5\%), compared with HBV HCC (19.6\%), HCV HCC (11.4\%), and alcoholic HCC (19.3\%).

\section{Comparisons of Clinical and Laboratory Variables}

Clinical and laboratory variables, including BMI, serum glucose, and total cholesterol levels are shown in table 3. There was no significant difference in mean BMI among HCC patients according to cause. Nearly one-third $(29.0 \%)$ of cHCC patients were overweight (BMI $\geq 25)$, but these rates were not significantly different from those with HCC from other causes. The proportion of $\mathrm{CHCC}$ patients who were obese (BMI $\geq 30$ ) was the highest $(3.7 \%)$ among HCC patients, but was not significantly different among the various patient groups. Hypertension was more prevalent in patients with cHCC than in patients with HBV HCC (49.4\% vs. 39.9\%, $\mathrm{p}=0.022)$ but not in patients with HCV HCC (53.9\%) or alcoholic HCC $(50.4 \%)$.

Hyperglycemia was more prevalent in patients with $\mathrm{CHCC}$ than in patients with HBV HCC $(45.7 \%$ vs. $32.3 \%$, p<0.001) but not in patients with HCV HCC (38.3\%) or alcoholic HCC (51.1\%). The mean total cholesterol levels were not significantly different among the HCC 


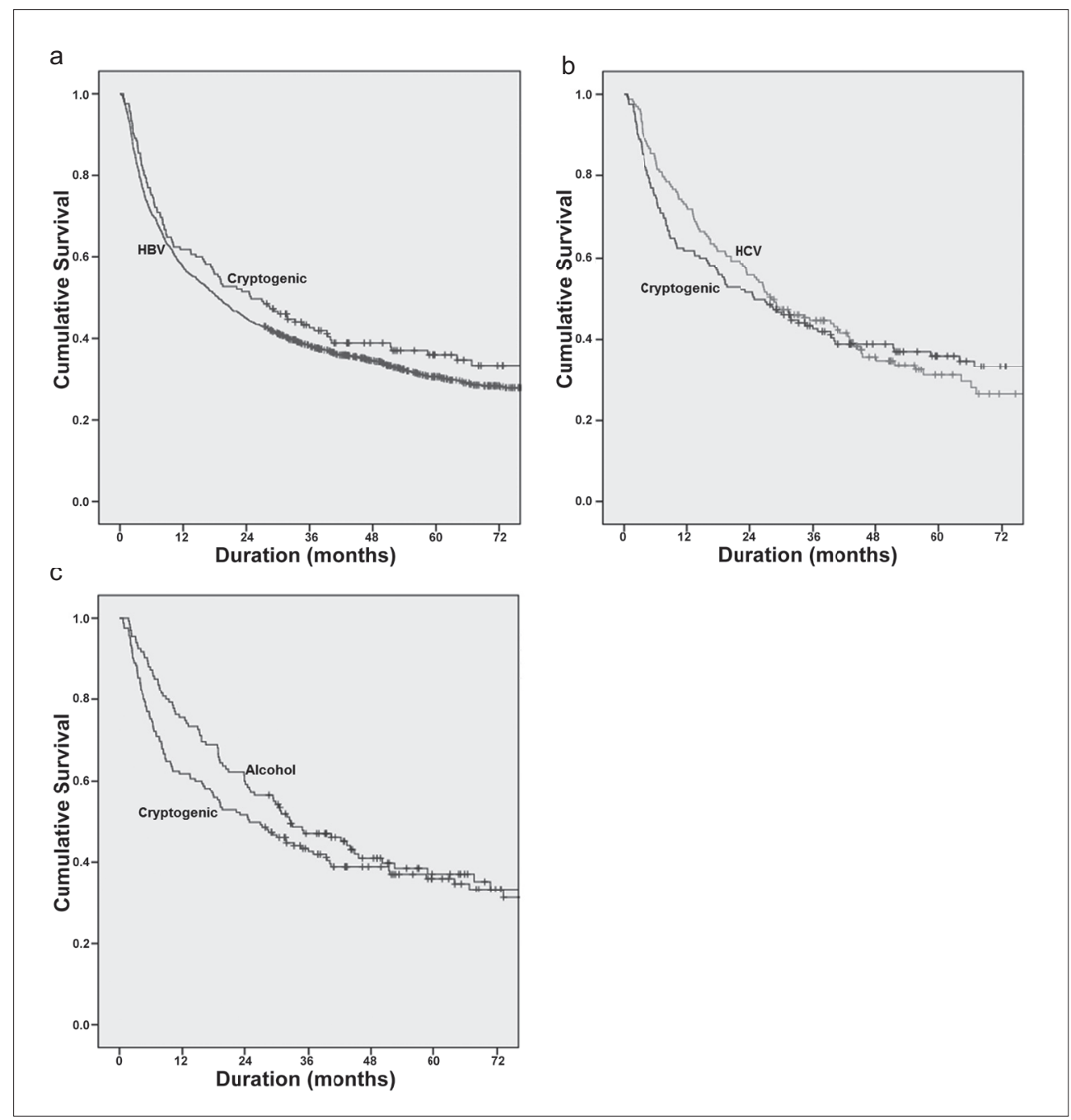

Fig. 1. Comparison of overall survival between cHCC and HBV HCC (a), HCV HCC (b), and alcoholic HCC (c). There were no significant differences between the groups in terms of survival rates (all p values $>0.05$ ).

groups; however, the incidence of hypercholesterolemia was higher in cHCC patients than in HCV HCC patients ( $4.9 \%$ vs. $0.6 \%, \mathrm{p}=0.018$ ).

Initial CT imaging studies revealed that fatty liver was more prevalent in patients with cHCC than in patients with HBV HCC $(26.5 \%$ vs. $19.4 \%$, p=0.038), but not for patients with HCV HCC (35.9\%) or alcoholic HCC (40.7\%).

The median AST value was significantly lower in patients with cHCC (48.0 IU/L) than in patients with HCC of viral-origin (HBV HCC, $59.0 \mathrm{IU} / \mathrm{L}$ and HCV HCC, $74.0 \mathrm{IU} / \mathrm{L}$, both $\mathrm{p}<0.001)$. Normal AST/ALT values were more frequent in patients with cryptogenic and alcoholic HCC. The median ALT value was also significantly lower in patients with cHCC (30.5 IU/L) than in patients with viral-origin HCC (HBV HCC, 41.0 IU/L and HCV HCC, 44.0 IU/L, both $\mathrm{p}<0.001$ ).

Comparisons According to the Presence of Anti-HBc Antibodies in cHCC Patients

Among cHCC patients, 137 (84.6\%) had anti-HBc antibodies. The cHCC patients without anti-HBc antibodies had better Eastern Cooperative Oncology Group (ECOG) performance, 


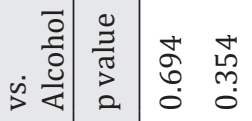

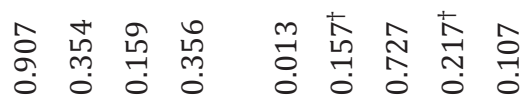

U্ত

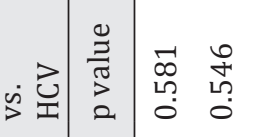

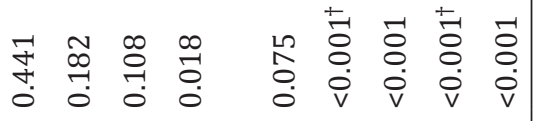

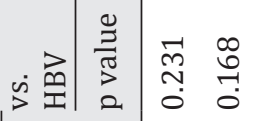

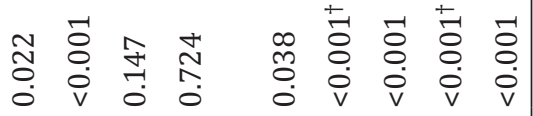


lower AFP levels compared to the group with anti-HBc antibodies (table 4). Interestingly, cHCC patients with anti-HBc antibodies were older, showed lower the MELD scores, AFP levels, lower AST/ALT levels, and a smaller proportion of patients with cirrhosis than patients with HBV HCC.

\section{Discussion}

cHCC comprised $9.1 \%$ of HCC cases in this study. The reported incidence of $\mathrm{cHCC}$, including NBNCNA HCC, in South Korea has increased from 2.3\% in 1993-1995 to 5.4\% in 2000-2002 ([22], and from 3.8\% in 2001-2005 to $12.2 \%$ in 2006-2010 [23]. In another study covering 2003-2012, cHCC comprised $6.8 \%$ of HCC cases [21]. The proportion of cHCC patients among all HCC patients is believed to be rising because of the increasing prevalence of NAFLD and the metabolic syndrome $[12,36]$.

In this study, cHCC patients had a poor median survival time (24.7 months). This fact could not be attributed to poor liver function, but could be attributable to tumor characteristics, i.e., large tumor size (mean size $7.4 \mathrm{~cm}$ ), poorly defined tumor type (34.0\%), portal vein invasion (30.4\%), and extrahepatic spread (19.1\%). Poor median survival could have resulted also from delay in making the diagnosis, as reflected in the lowest rate $6.8 \%$ of mUICC stage I tumors among the four kinds of HCC studied. Perhaps patients with NAFLD, the putative major cause of cHCC, had fewer symptoms than patients with chronic HBV/ HCV hepatitis or alcoholic liver diseases, and therefore less frequently received liver screening [37]. Thus, the diagnosis of cHCC might be delayed, meaning that the tumors are larger and more aggressive than other HCCs when discovered. Nonetheless, cHCC patients had better survival than HBV HCC patients (HR 1:1.251) according to multivariate analysis. Consequently, patients with NAFLD should undergo regular surveillance for the development of HCC. In addition, the proportion of cHCC patients with AFP values $>200 \mathrm{ng} / \mathrm{mL}$ was the second highest, which might result from the larger size and more aggressive clinical characteristics of cHCC at diagnosis.

cHCC patients in the current study had the lowest prevalence of cirrhosis in initial imaging studies, and all HCC patients showed a tendency toward a lower prevalence of cirrhosis regardless of cause compared to that found in other studies [38]. These results might be caused by ambiguity of the clinical definition of cirrhosis and limitations in the radiological diagnosis of cirrhosis, including bias in individual radiologist's readings. To compensate for these weaknesses, we adopted the APRI score for analyzing the severity of liver fibrosis. According to the APRI scores, cHCC patients had less liver fibrosis than patients with viralinduced HCC, but more than patients with alcoholic HCC. The high mean platelet count of cHCC patients also may reflect lower levels of liver fibrosis.

In this study, the proportion of female patients was highest $(22.8 \%)$ in the cHCC group, and was significantly higher than that in HBV HCC patients (16.1\%). This might be related to the higher prevalence of NAFLD in older women [39]. However, the relationship between gender and the etiology of HCC needs further evaluation.

The proportion of obese (BMI $\geq 30$ ) patients was higher for cHCC than for the other types of HCC, but the difference was not statistically significant. The incidence of metabolic syndrome was similar for cryptogenic, HCV, and alcoholic HCC. HCV and alcoholic HCC are often accompanied by the metabolic syndrome, which can obscure the causal differences between the various kinds of HCC [40]. In our study, the proportion of overweight (BMI $\geq 25$ ) patients with cHCC was lower than that in other reports [21, 24]. This discrepancy may be attributable to differences in the proportion of patients with advanced HCC and overlapping 


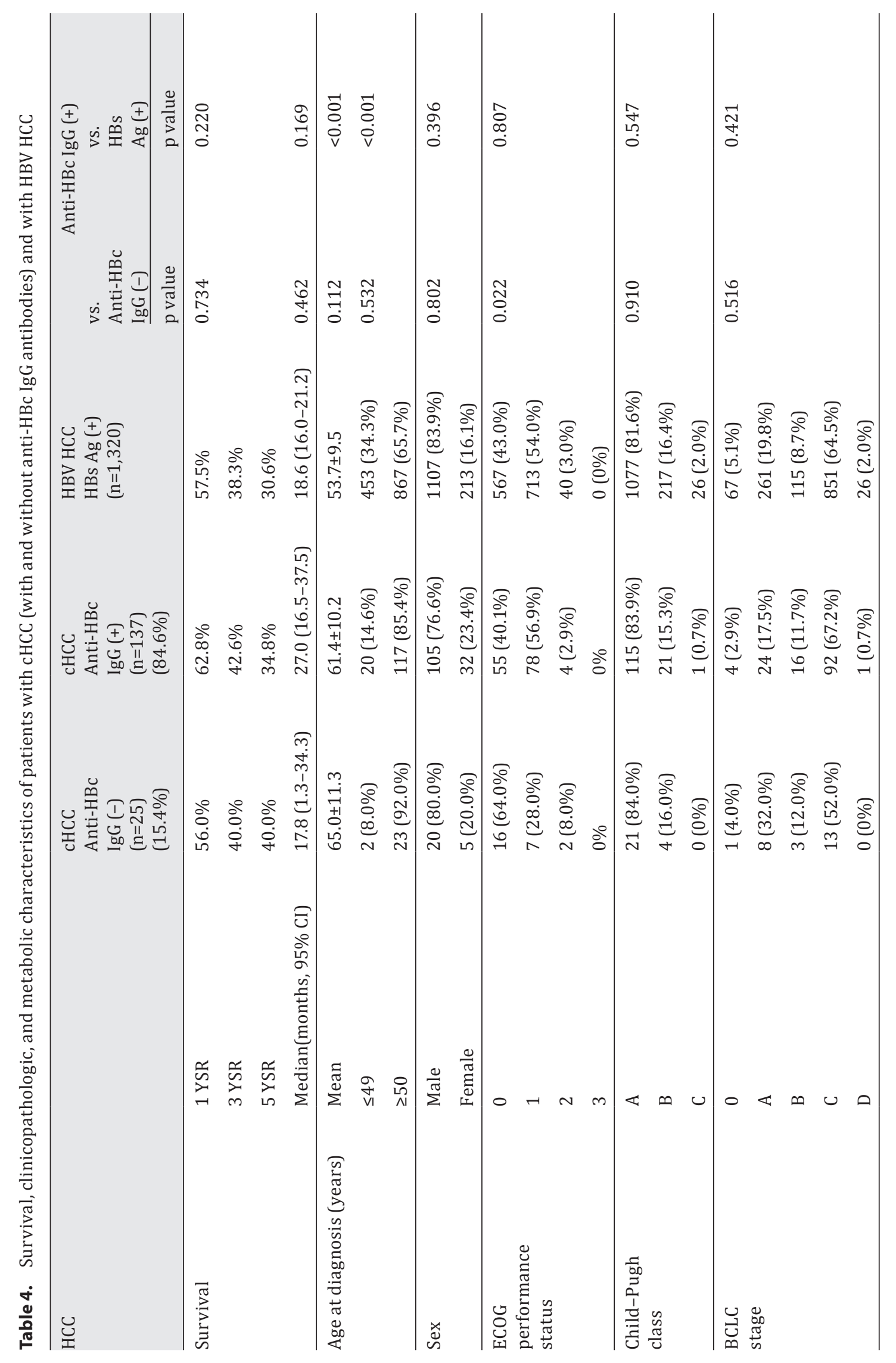




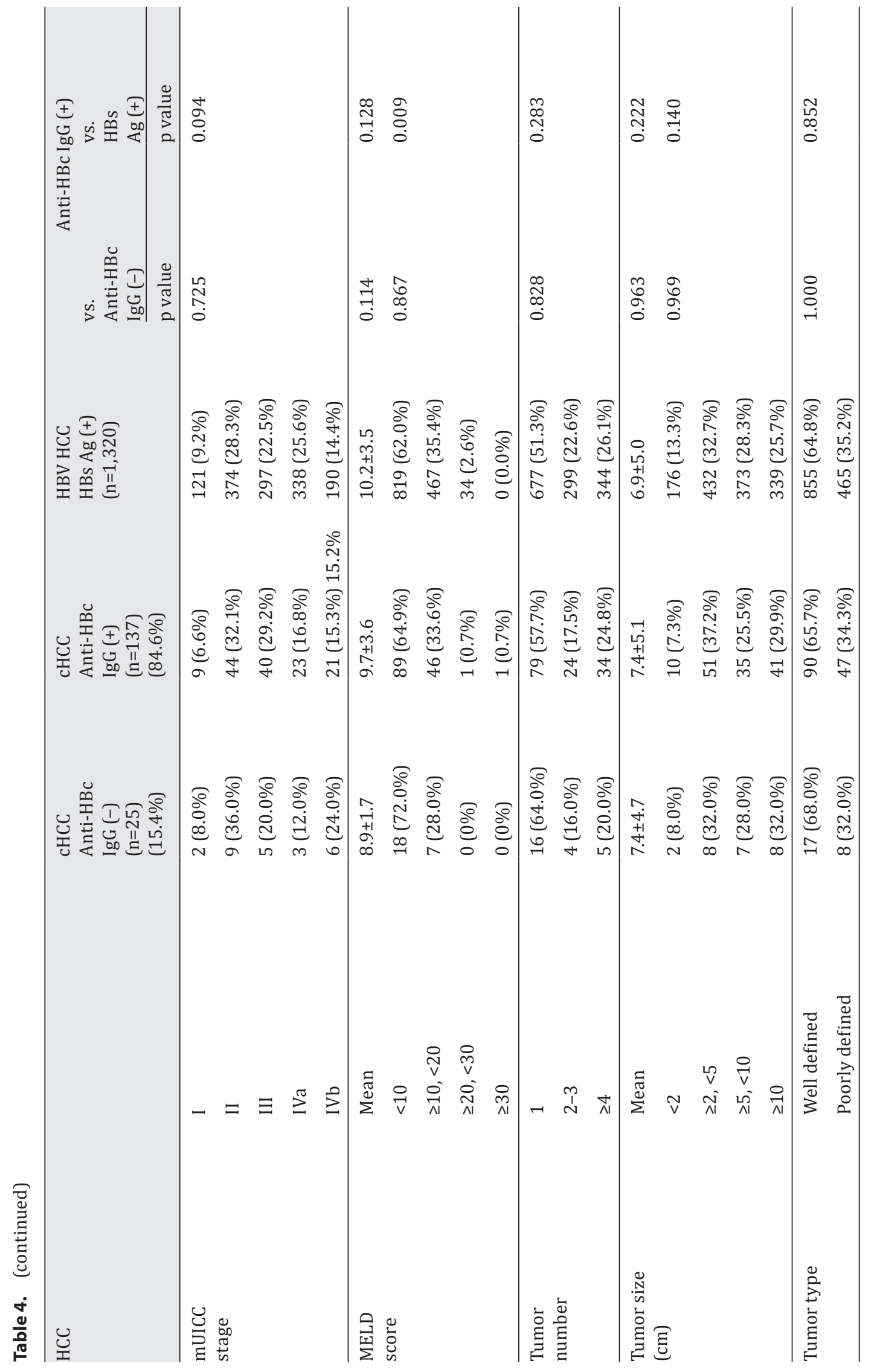




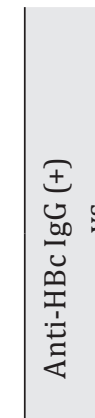

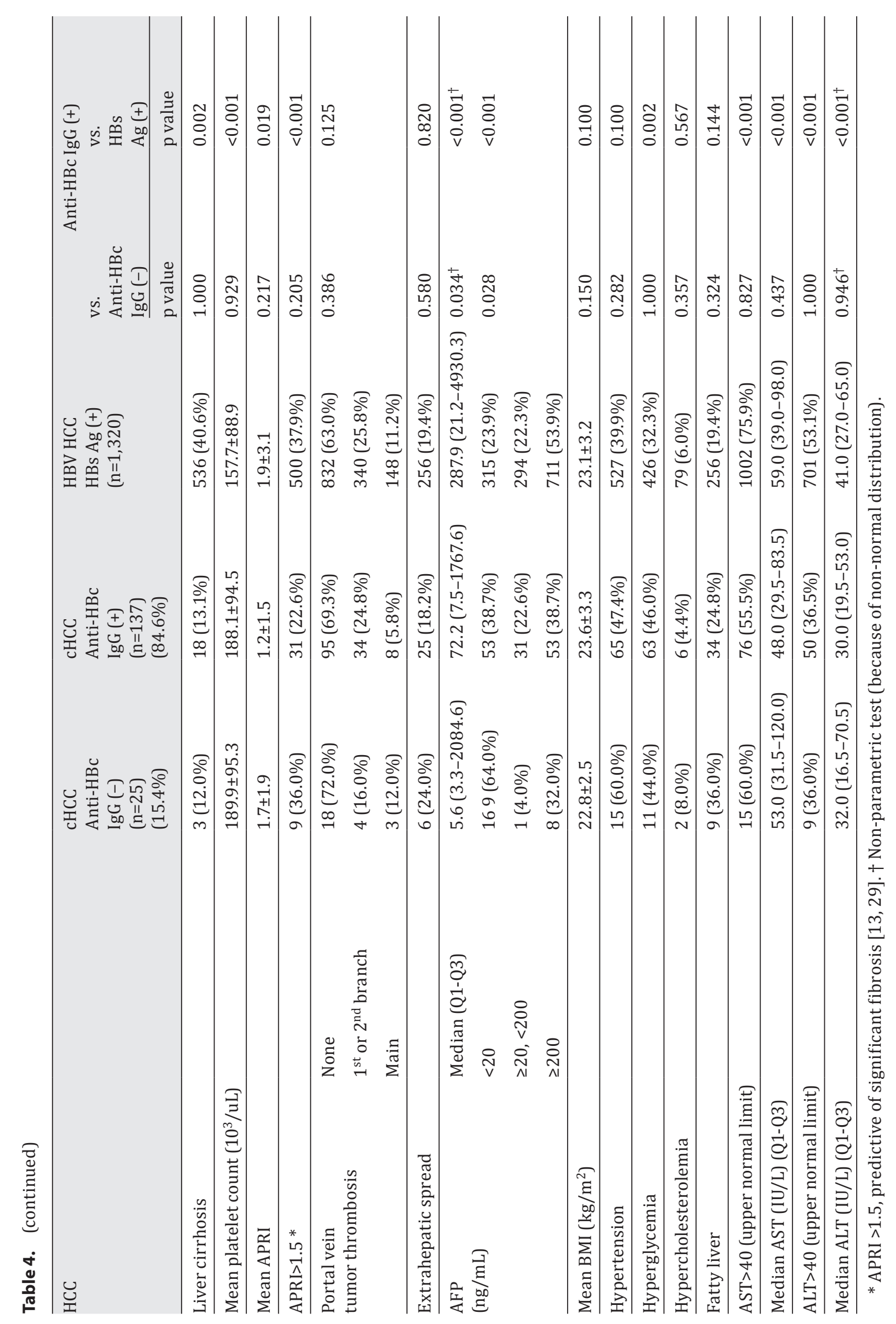


occurrence of metabolic syndrome in the study population. Unfortunately, the inherent limitations of our study (because of its retrospective nature) meant that we did not have information regarding the history of obesity and changes in weight after the development of HCC.

In comparison to other reports [18, 41,42], our patients had a lower prevalence of diabetes, which may be related to the low prevalence of cirrhosis in our study. A Japanese study also reported that underlying cirrhosis affects the prevalence of diabetes in patients with NAFLD or HCV [43].

The proportion of cHCC patients with hypertension and fatty liver cHCC was higher than that for HBV HCC, but not for HCV HCC or alcoholic HCC. One possible reason is that patients with HCV or alcoholic HCC are likely to have concurrent metabolic syndrome, as mentioned above. In other reports also, the proportion of cHCC patients with hypertension was higher than that of HBV HCC patients [21,24]. Depending on the definition of hypertension, the proportion of hypertensive patients will change; nonetheless, we could determine the relative proportions of patients with hypertension for HCC with different causes.

The fact that HBV is endemic in Korea could be the reason for the presence of anti-HBc antibodies in so many patients with cHCC. It was considered that the presence of anti-HBc antibodies might modify the characteristics of HCC toward those of HBV HCC. However, analysis of data for CHCC patients with and without anti-HBc antibodies showed significant differences between HBV HCC and CHCC with anti-HBc antibodies. According to the protocol of this study, if non-HCV, non-alcoholic patients did not have HBsAg, regardless the presence of anti-HBc antibodies, they were classified as having cHCC. However, many patients with cHCC had anti-HBc antibodies, and these patients showed some tumor characteristics intermediate between those of HBV HCC patients and those of "pure" cHCC patients.

This cohort study had several limitations. First, cHCC in South Korea includes HCC from causes other than NAFLD, such as a past HBV infection and unknown causes. The possible role of past HBV infections is uncertain in CHCC patients with anti-HBc antibodies. Second, patient histories of alcohol consumption were obtained through self-reporting, which may not be reliable. It is possible that the proportion of patients who had alcoholic liver disease as the cause of HCC was underestimated [25], and this underestimation could have affected the observed clinical features of the population classified as cHCC. Third, metabolic factors resulting from the metabolic syndrome were not clearly implicated as the main cause of cHCC in this study. Values of BMI, hypertension, and hyperglycemia obtained only from the initial questionnaire and initial laboratory studies could contain false negatives or positives. Finally, the diagnosis of cirrhosis or fatty liver was made based on the initial CT imaging studies only. However, bias from individual radiologists may have skewed and underestimated the results.

In summary, cHCC comprised $9.1 \%$ of HCC patients. The median survival times of cHCC was the second poorest after HBV HCC. cHCC patients had the largest tumors and the second highest proportion of poor prognostic factors such as the proportion of poorly defined tumors and extrahepatic spread in imaging studies, but had the lowest prevalence of cirrhosis on initial imaging studies. cHCC patients were shown by multivariate analysis to have better survival than HBV HCC patients. This study did not find conclusive evidence of the effects of clinical and laboratory variables such as BMI, glucose, and cholesterol levels in our population, likely because HCV HCC and alcoholic HCC could be affected by comorbid metabolic syndrome. cHCC patients had lower AST/ALT levels than those with viral-induced HCC. Most patients with $\mathrm{cHCC}$ had anti-HBc antibodies, but we could not determine whether the presence of anti-HBc antibodies affected clinical characteristics. It is hoped that this study will contribute to understanding of the behavior and appropriate management of cHCC in HBVendemic areas such as South Korea. 


\section{Acknowledgments}

The authors thank R.N. Eun-Ah Cho, R.N. Da In Shin, and R.N. Hye Ran Jung for collecting the raw data. This study was supported by a grant from the National Cancer Center, Korea (\#1510520-1)

\section{Disclosures}

H.-W. Kwak, J.-W. Park, YH Koh, JH Lee, A. Yu, and B.-H. Nam have no conflicts of interest or financial funding to report.

\section{References}

1 Ohnishi K, Iida S, Iwama S, Goto N, Nomura F, Takashi M, Mishima A, Kono K, Kimura K, Musha H, Kotota K, Okuda K: The effect of chronic habitual alcohol intake on the development of liver cirrhosis and hepatocellular carcinoma: relation to hepatitis B surface antigen carriage. Cancer 1982;49:672-677.

2 Donato F, Tagger A, Chiesa R, Ribero ML, Tomasoni V, Fasola M, Gelatti U, Portera G, Boffetta P, Nardi G: Hepatitis B and C virus infection, alcohol drinking, and hepatocellular carcinoma: a case-control study in Italy. Brescia HCC Study. Hepatology 1997;26:579-584.

3 Harrison SA, Torgerson S, Hayashi PH: The natural history of nonalcoholic fatty liver disease: a clinical histopathological study. Am J Gastroenterol 2003;98:2042-2047.

4 Bugianesi E, Leone N, Vanni E, Marchesini G, Brunello F, Carucci P, Musso A, De Paolis P, Capussotti L, Salizzoni M, Rizzetto M: Expanding the natural history of nonalcoholic steatohepatitis: from cryptogenic cirrhosis to hepatocellular carcinoma. Gastroenterology 2002;123:134-140.

5 Marrero JA, Fontana RJ, Su GL, Conjeevaram HS, Emick DM, Lok AS: NAFLD may be a common underlying liver disease in patients with hepatocellular carcinoma in the United States. Hepatology 2002;36:13491354.

6 El-Serag HB, Rudolph KL: Hepatocellular carcinoma: epidemiology and molecular carcinogenesis. Gastroenterology 2007;132:2557-2576.

7 Starley BQ, Calcagno CJ, Harrison SA: Nonalcoholic fatty liver disease and hepatocellular carcinoma: a weighty connection. Hepatology 2010;51:1820-1832.

8 Baffy G, Brunt EM, Caldwell SH: Hepatocellular carcinoma in non-alcoholic fatty liver disease: an emerging menace. J Hepatol 2012;56:1384-1391.

9 Tsukuma H, Hiyama T, Tanaka S, Nakao M, Yabuuchi T, Kitamura T, Nakanishi K, Fujimoto I, Inoue A, Yamazaki $\mathrm{H}$, et al: Risk factors for hepatocellular carcinoma among patients with chronic liver disease. $\mathrm{N}$ Engl J Med 1993;328:1797-1801.

10 Siegel R, Naishadham D, Jemal A: Cancer statistics, 2012. CA Cancer J Clin 2012;62:10-29.

11 Yang JD, Roberts LR: Hepatocellular carcinoma: A global view. Nat Rev Gastroenterol Hepatol 2010;7:448458.

12 Adams LA, Lindor KD: Nonalcoholic fatty liver disease. Ann Epidemiol 2007;17:863-869.

13 Kawamura Y, Arase Y, Ikeda K, Seko Y, Imai N, Hosaka T, Kobayashi M, Saitoh S, Sezaki H, Akuta N, Suzuki F, Suzuki Y, Ohmoto Y, Amakawa K, Tsuji H, Kumada H: Large-scale long-term follow-up study of Japanese patients with non-alcoholic Fatty liver disease for the onset of hepatocellular carcinoma. Am J Gastroenterol 2012;107:253-261.

14 Younossi ZM, Stepanova M, Afendy M, Fang Y, Younossi Y, Mir H, Srishord M: Changes in the prevalence of the most common causes of chronic liver diseases in the United States from 1988 to 2008. Clin Gastroenterol Hepatol 2011;9:524-530 e1; quiz 60.

15 Chitturi S, Farrell GC, Hashimoto E, Saibara T, Lau GK, Sollano JD, Asia-Pacific Working Party on NAFLD: Non-alcoholic fatty liver disease in the Asia-Pacific region: definitions and overview of proposed guidelines. J Gastroenterol Hepatol 2007;22:778-787.

16 Amarapurkar DN, Hashimoto E, Lesmana LA, Sollano JD, Chen PJ, Goh KL, Asia-Pacific Working Party on NAFLD: How common is non-alcoholic fatty liver disease in the Asia-Pacific region and are there local differences? J Gastroenterol Hepatol 2007;22:788-793.

17 Yang JD, Harmsen WS, Slettedahl SW, Chaiteerakij R, Enders FT, Therneau TM, et al: Factors that affect risk for hepatocellular carcinoma and effects of surveillance. Clin Gastroenterol Hepatol 2011;9:617-623. e1.

18 Yatsuji S, Hashimoto E, Tobari M, Taniai M, Tokushige K, Shiratori K: Clinical features and outcomes of cirrhosis due to non-alcoholic steatohepatitis compared with cirrhosis caused by chronic hepatitis C. J Gastroenterol Hepatol 2009;24:248-254.

19 Toshikuni N, Izumi A, Nishino K, Inada N, Sakanoue R, Yamato R, Suehiro M, Kawanaka M, Yamada G: Comparison of outcomes between patients with alcoholic cirrhosis and those with hepatitis $\mathrm{C}$ virus-related cirrhosis. J Gastroenterol Hepatol 2009;24:1276-1283. 
20 Fattovich G, Pantalena M, Zagni I, Realdi G, Schalm SW, Christensen E, European Concerted Action on Viral Hepatitis (EUROHEP): Effect of hepatitis B and C virus infections on the natural history of compensated cirrhosis: a cohort study of 297 patients. Am J Gastroenterol 2002;97:2886-2895.

21 Lee SS, Jeong SH, Byoun YS, Chung SM, Seong MH, Sohn HR, Min BY, Jang ES, Kim JW, Park GJ, Lee YJ, Lee $\mathrm{KH}, \mathrm{Ahn} \mathrm{S}$ : Clinical features and outcome of cryptogenic hepatocellular carcinoma compared to those of viral and alcoholic hepatocellular carcinoma. BMC Cancer 2013;13:335.

22 Oh KC, Park SH, Park JC, Jin DK, Park CS, Kim KO, Jang HJ, Lee JY, Park CH, Han TH, Yoo KS, Kim JH, Kim DJ, Lee MS, Park CK: [Is the prevalence of cryptogenic hepatocellular carcinoma increasing in Korea?] Korean J Gastroenterol 2005;45:45-51.

23 Cho EJ, Kwack MS, Jang ES, You SJ, Lee JH, Kim YJ, Yoon JH, Lee HS: Relative etiological role of prior hepatitis B virus infection and nonalcoholic fatty liver disease in the development of non-B non-C hepatocellular carcinoma in a hepatitis B-endemic area. Digestion 2011;84(Suppl 1):17-22.

24 Rim MY, Kwon OS, Ha M, Kim JS, Ko KI, Kim DK, Jang PK, Han JY, Park PH, Jung YK, Choi DJ, Kim YS, Kim JH: [Clinical features of non-alcoholic fatty liver disease in cryptogenic hepatocellular carcinoma]. Korean J Gastroenterol 2014;63:292-298.

25 Kwak HW, Park JW, Nam BH, Yu A, Woo SM, Kim TH, et al: Clinical outcomes of a cohort series of patients with hepatocellular carcinoma in a hepatitis B virus-endemic area. J Gastroenterol Hepatol 2014;29: 820829.

26 Park JW; Korean Liver Cancer Study Group and National Cancer Center: [Practice guideline for diagnosis and treatment of hepatocellular carcinoma]. Korean J Hepatol 2004;10:88-98.

27 Korean Liver Cancer Study Group and National Cancer Center: [Practice guidelines for management of hepatocellular carcinoma 2009]. Korean J Hepatol 2009;15:391-423.

28 Simonetti J, Bulkow L, McMahon BJ, Homan C, Snowball M, Negus S, Williams J, Livingston SE: Clearance of hepatitis B surface antigen and risk of hepatocellular carcinoma in a cohort chronically infected with hepatitis B virus. Hepatology 2010;51:1531-1537.

29 Ueno S, Tanabe G, Nuruki K, Hamanoue M, Komorizono Y, Oketani M, et al: Prognostic performance of the new classification of primary liver cancer of Japan (4th edition) for patients with hepatocellular carcinoma: a validation analysis. Hepatol Res 2002;24:395-403.

30 Llovet JM, Brú C, Bruix J: Prognosis of hepatocellular carcinoma: the BCLC staging classification. Semin Liver Dis 1999;19:329-338.

31 Kamath PS, Wiesner RH, Malinchoc M, Kremers W, Therneau TM, Kosberg CL, D’Amico G, Dickson ER, Kim WR: A model to predict survival in patients with end-stage liver disease. Hepatology 2001;33:464-470.

32 Ucar F, Sezer S, Ginis Z, Ozturk G, Albayrak A, Basar O, Ekiz F, Coban S, Yuksel O, Armutcu F, Akbal E: APRI, the FIB-4 score, and Forn's index have noninvasive diagnostic value for liver fibrosis in patients with chronic hepatitis B. Eur J Gastroenterol Hepatol 2013;25:1076-1081.

33 Report of the Expert Committee on the Diagnosis and Classification of Diabetes Mellitus. Diabetes Care 1997;20:1183-1197.

34 The Expert Panel: Report of the National Cholesterol Education Program Expert Panel on Detection, Evaluation, and Treatment of High Blood Cholesterol in Adults. Arch Intern Med 1988;148:36-69.

35 Shin WG, Park SH, Jun SY, Jung JO, Moon JH, Kim JP, Kim KO, Park CH, Hahn TH, Yoo KS, Kim JH, Park CK: Simple tests to predict hepatic fibrosis in nonalcoholic chronic liver diseases. Gut Liver 2007;1:145-150.

36 Siegel AB, Zhu AX: Metabolic syndrome and hepatocellular carcinoma: two growing epidemics with a potential link. Cancer 2009;115:5651-5661.

37 Hashimoto E, Tokushige K: Prevalence, gender, ethnic variations, and prognosis of NASH. J Gastroenterol 2011;46(Suppl 1):63-69.

38 White DL, Kanwal F, El-Serag HB: Association between nonalcoholic fatty liver disease and risk for hepatocellular cancer, based on systematic review. Clin Gastroenterol Hepatol 2012;10:1342-1359 e2.

39 Kojima S, Watanabe N, Numata M, Ogawa T, Matsuzaki S: Increase in the prevalence of fatty liver in Japan over the past 12 years: analysis of clinical background. J Gastroenterol 2003;38:954-961.

40 Li WC, Lee YY, Chen IC, Sun C, Chiu FH, Chuang CH: Association between the hepatitis B and C viruses and metabolic diseases in patients stratified by age. Liver Int 2013;33:1194-1202.

41 Ascha MS, Hanouneh IA, Lopez R, Tamimi TA, Feldstein AF, Zein NN: The incidence and risk factors of hepatocellular carcinoma in patients with nonalcoholic steatohepatitis. Hepatology 2010;51:1972-1978.

42 Bhala N, Angulo P, van der Poorten D, Lee E, Hui JM, Saracco G, Adams LA, Charatcharoenwitthaya P, Topping JH, Bugianesi E, Day CP, George J: The natural history of nonalcoholic fatty liver disease with advanced fibrosis or cirrhosis: an international collaborative study. Hepatology 2011;54:1208-1216.

43 Arase Y, Kobayashi M, Suzuki F, Suzuki Y, Kawamura Y, Akuta N, Imai N, Kobayashi M, Sezaki H, Matsumoto N, Saito S, Hosaka T, Ikeda K, Kumada H, Ohmoto Y, Amakawa K, Hsieh SD, Ogawa K, Tanabe M, Tsuji $\mathrm{H}$, Kobayashi T: Difference in malignancies of chronic liver disease due to non-alcoholic fatty liver disease or hepatitis C in Japanese elderly patients. Hepatol Res 2012;42:264-272. 